\title{
Reorganização da Rede de Atenção à Saúde para o enfrentamento da COVID-19 no município de Canaã dos Carajás, Pará
}

\author{
Eliana Pessoa do Vale, Gizele Moreira Rodrigues, Douglas Pacheco da Costa, Juliana da Mota \\ Queiroz, Débora Garcia Lima, Luanna Priscylla Faustino Medeiros, Daiane Celestrini Oliveira, \\ Eric Gonçalves Baia, Ana Lúcia Araújo Costa, Miguel Bento Pereira Neto, Caio Túlio Vale \\ Frazão
}

\section{RESUMO}

É urgente a necessidade de reorganizar a Rede de Atenção à Saúde integrando os diversos pontos e tipos de serviço para o enfrentamento da COVID-19. OBJETIVO: Descrever a reorganização da Rede de Atenção à Saúde (RAS) para o enfrentamento da COVID-19 no munícipio de Canaã dos Carajás, no Pará. MÉTODO: Trata-se de um estudo do tipo relato de experiência da reorganização da RAS do município de Canaã dos Carajás, os dados aqui relatados são baseados nos documentos oficiais produzidos no município e no relato da gestão. RESULTADOS: Reorganização do acesso com ampliação da carteira de serviços da Atenção Básica $(A B)$, elaboração e readequação de novos serviços para o fortalecimento da RAS e reorganização da Assistência Hospitalar. CONCLUSÃO: Os processos de planejamento, monitoramento, avaliação e reorganização das RAS devem ser contínuos durante a pandemia, sempre levando em conta as evidências epidemiológicas locais para traçar novas estratégias para garantia de um sistema de saúde universal e equânime.

Palavras-chave: Pandemias; Infecções por Coronavírus; Atenção Primária à Saúde; Sistema Único de Saúde.

\section{ABSTRACT}

There is an urgent need to reorganise the Health Care Network to integrate the different points of service to handle COVID-19. OBJECTIVE: To describe the Reorganisation of the Health Care Network (RAS) to confront COVID-19 in the municipality of Canaã dos Carajás in Pará. METHOD: This is a study of the type of experience report of the RAS Reorganisation in the municipality of Canaã dos Carajás, the data reported in this article are based on official documents produced by and on the municipality's management report. RESULTS: Reorganisation of access and expansion of the ABS service portfolio, development of new services to strengthen the $\mathrm{HCN}$ and reorganisation of Hospital Assistance. CONCLUSION: The planning, monitoring, evaluation and reorganisation processes of the RAS must be continuous during the pandemic, always taking into account the local epidemiological evidence to outline new strategies to guarantee a universal and equitable health system.

Keywords: Pandemics; Coronavirus Infections; Primary Health Care; Unified Health System.
Revista da Rede APS 2020

Publicada em: 09/06/2020

DOI:10.14295/aps.v2i2.101

Eliana Pessoa do Vale Gizele Moreira Rodrigues Douglas Pacheco da Costa Juliana da Mota Queiroz Débora Garcia Lima Luana Priscylla F. Medeiros Daiane Celestrini Oliveira Eric Gonçalves Baia Ana Lúcia A. Costa Miguel Bento P. Neto Caio Túlio V. Frazão. (Secretaria Municipal de Saúde (SEMSA); Canaã dos Carajás, PA, Brasil)

Correspondência para: Eliana Pessoa do Vale elianapvv@hotmail.com

Gizele Moreira Rodgrigues gizelhi rodrigues@hotmail.com

Douglas Pacheco da Costa douglaspcosta@hotmail.com 


\section{INTRODUÇÃO}

A COVID-19 é uma doença causada pelo agente etiológico SARS-CoV-2 e suas manifestações podem variar de infecções assintomáticas a quadros graves ${ }^{1}$. A elevada transmissibilidade do SARS-CoV-2 faz com que o crescimento do número de casos seja exponencial. No Brasil, o primeiro caso foi confirmado em 26 de fevereiro de 2020 relativo a um usuário proveniente de uma viagem à Europa. A partir de março, vários casos foram confirmados em diferentes regiões do país². Em 20 de março, a Portaria № 454 do Ministério da Saúde declarou a transmissão comunitária do coronavírus (COVID-19) em todo território nacional e a necessidade de unir os esforços para reduzir a transmissibilidade e oportunizar manejo adequado dos casos leves na Rede de Atenção Básica à Saúde e dos casos graves na rede de urgência/emergência e hospitalar ${ }^{3}$.

Essa elevação dos casos em um curto espaço de tempo causa alto impacto na saúde pública, demostrando a necessidade da atuação rápida do Sistema Único de Saúde (SUS) e saúde suplementar. Nesse sentido, faz-se necessária a elaboração de planos para o enfrentamento da pandemia e a reorganização das práticas de saúde para promover assistência rápida aos usuários, por meio de ações de prevenção e promoção, tratamento, controle, reabilitação e cura para garantir e alcançar os princípios da universalidade, a integralidade e a equidade no enfrentamento da COVID-194,5.

Durante a pandemia, a Atenção Básica tem papel fundamental como porta de entrada do Sistema Único de Saúde, oferecendo um atendimento resolutivo com os casos leves e identificação precoce dos casos graves que devem ser encaminhados para serviços especializados, mantendo a coordenação do cuidado em todos os níveis da atenção em saúde ${ }^{6}$.

É urgente a necessidade de reorganizar a Rede de Atenção à Saúde (RAS) integrando os diversos pontos do serviço. De acordo com as estimativas oficiais, a Atenção Básica pode manejar $81 \%$ dos casos de COVID-19, 14\% vão precisar de internação hospitalar e 5\% demandarão leitos de UTI. A pandemia demostrou a real necessidade de um sistema de saúde integrado em forma de RAS, por meio da organização dos pontos de atenção definindo os papéis e fluxos para o atendimento dos sintomáticos respiratórios e dos usuários que apresentem as mais diversas necessidades de saúde, evidenciando a necessidade do aperfeiçoamento dos mecanismos da RAS para - alcance da integralidade do cuidado, perpassando do trabalho do Agente Comunitário de Saúde da equipe da APS até o atendimento de um profissional da UTI ${ }^{1}$. O presente relato de caso visa descrever a reorganização da RAS para o enfrentamento da COVID-19 no munícipio de Canaã dos Carajás no Pará.

\section{MÉTodo}

Trata-se de um estudo do tipo relato de experiência da reorganização da RAS do Município de Canaã dos Carajás, localizado no interior do estado do Pará. Possui a população estimada de 44.569 habitantes pelo IBGE e 58.830 pessoas cadastradas no e-SUS. O município possui extensão territorial de $3.146,407 \mathrm{~km}^{2}$, densidade demográfica de 8,49 habitantes $/ \mathrm{km}^{2}$ e Índice de Desenvolvimento Humano (IDH) de 0,673. A maioria da população possui entre 25 e 44 anos e os trabalhadores possuem salário médio mensal de 4.9 salários mínimos. Além disso, $100 \%$ dos domicílios possuem esgoto sanitário adequado, $11,49 \%$ da população é considerada em extrema pobreza e $56,7 \%$ dos habitantes possuem plano de saúde ${ }^{6}$.

Canaã dos Carajás possui um hospital municipal com 51 leitos, sendo onze de observação na emergência e três leitos de pré-parto. A zona urbana do município possui nove Equipes de Saúde da Família divididas em sete unidades. Cada equipe de Saúde da Família conta com uma equipe de Saúde Bucal e o município conta com 
um Núcleo de Apoio à Saúde da Família (NASF). A Zona Rural conta com quatro unidades de saúde, duas de ESF e dois PACS, possuindo 100\% de cobertura de Estratégia Saúde da Família ${ }^{7}$.

Entre os anos de 2017 a 2019, o município, em parceria com o projeto Ciclo Saúde, realizou a reorganização da Atenção Básica à Saúde por meio do acolhimento à demanda espontânea durante todo o horário de funcionamento e implantação do Prontuário Eletrônico Integrado, fortalecendo a Atenção Primária como porta de entrada, com garantia do acesso e horário estendido, proporcionando maior resolutividade. A assistência se tornou mais satisfatória por meio do primeiro contato com o ACS do "posso ajudar" que realiza o acolhimento com escuta qualificada, e do enfermeiro que atua na classificação de risco e de vulnerabilidade. A reorganização da APS em Canaã dos Carajás fortaleceu o vínculo entre usuários e equipe, reorganizou o processo de trabalho, que proporcionou um atendimento mais humanizado, garantindo a equidade, a integralidade, a longitudinalidade, a universalidade, a coordenação do cuidado e a redução das barreiras de acesso ${ }^{7}$.

Em 2020, frente aos desafios postos pela pandemia, foi necessário remodelar toda a RAS para poder prestar a melhor assistência aos usuários com sintomas respiratórios e também para os demais usuários da RAS. Canaã dos Carajás faz parte da rede de 32 municípios que contam com o apoio do projeto Ciclo Saúde COVID-19, iniciativa da Fundação Vale em parceria intersetorial com o Centro de Promoção da Saúde (CEDAPS) e o Programa de Pós-Graduação da Universidade Estácio de Sá, que atuam por meio da disponibilidade de apoio técnico à gestão, doações de kit COVID-19 com materiais e insumos para a $A B$, pesquisa $e$ produção de informativos com protocolos e fluxos sobre a COVID-19 e sobre as linhas de cuidado da $A B$.

O relato foi baseado na experiência da gestão do município de Canaã dos Carajás e no apoio à gestão do Projeto Ciclo Saúde durante o período de fevereiro a maio de 2020. Os dados aqui relatados são baseados nos documentos oficiais produzidos pelo município como o Plano de Contingência, fluxos de atendimento da rede e no relato da gestão do município.

\section{RESULTADOS}

Em fevereiro de 2020, quando foi confirmado o primeiro caso de COVID-19 no país, o município instituiu o Centro de Operação de Emergência em Saúde (COES) visando promover a resposta coordenada por meio da articulação e da integração dos atores envolvidos e da análise dos dados e das informações para subsidiar a tomada de decisão dos gestores. Também foi elaborado o Plano de Contingência Municipal, no qual foram definidos os níveis de resposta e os setores envolvidos na organização da RAS para o enfrentamento da COVID-19. A reorganização foi realizada por meio de três estratégias: reorganização do acesso e ampliação da carteira de serviços da $A B$, elaboração de novos serviços para o fortalecimento da RAS e a reorganização da Assistência Hospitalar.

As estratégias visam proporcionar a ampliação do acesso dos sintomáticos respiratórios leves, manutenção do cuidado dos doentes crônicos, fortalecimento da rede, reorganização do hospital municipal, ampliação do horário de funcionamento das unidades, testagem ampliada dos sintomáticos respiratórios e fortalecimento da assistência hospitalar dos casos graves. Além das três estratégias de reorganização da RAS, também foram implementadas medidas de precaução comunitária e de proteção dos profissionais.

\section{1 - Reorganização do acesso e ampliação da carteira de serviços da $A B$}

\section{1 - Porta de entrada dos sintomáticos respiratórios}

Para o fortalecimento da $A B$ como porta de entrada do sistema foi instituída uma unidade de referência para $o$ atendimento dos 
sintomáticos respiratórios. A unidade teve o horário de funcionamento ampliado, passando a atender os usuários 24 horas por dia, incluindo os finais de semana e feriados. Foram contratados mais profissionais e adquiridos novos equipamentos para estruturar a unidade para os atendimentos dos sintomáticos respiratórios leves e também do primeiro atendimento para os casos com sinais de agravamento. Foi estruturada uma sala de procedimento e uma sala de observação com leitos onde o usuário pode ficar até 12 horas em observação para estabilização e liberação para o isolamento domiciliar ou transferência para internação no hospital de campanha. A unidade também realiza exames laboratoriais complementares e quando necessário RX, o usuário é levado de ambulância para realizar o exame.

O primeiro contato com o usuário é realizado por uma equipe multiprofissional de nível superior, utilizando máscara cirúrgica, que identifica o motivo da consulta. Os usuários sem queixas respiratórias são orientados em relação ao novo fluxo da unidade. Caso seja identificada a necessidade de atendimento de demanda espontânea, demanda programada ou atendimentos odontológicos de emergência, o usuário será encaminhado para as Unidades de Saúde que estão realizando os atendimentos dos usuários com outros sintomas e das linhas de cuidado.

Todo usuário que apresenta sintomas gripais é abordado como caso suspeito de COVID-19 e será notificado. O profissional de saúde responsável pelo "posso ajudar" entregam a máscara e orienta sobre seu uso correto e a higiene das mãos. O usuário é informado sobre o atendimento do DISK CORONAVÍRUS atendimento 0800 do município para tirar dúvidas e realizar o teleatendimento dos usuários com sintomas leves.

Como medidas de engenharia para proteção dos profissionais e usuários, ainda na sala de espera que foi montada na área externa da unidade, os usuários devem se manter a 1 metro de distância e utilizar a máscara durante todo o tempo. Só é permitida a entrada na unidade durante o atendimento os acompanhantes de crianças e idosos, já os demais acompanhantes são aconselhados a aguardar nas cadeiras instaladas na área externa da unidade.

Os usuários são encaminhados para o acolhimento e é realizada a escuta qualificada por um dos integrantes da equipe multiprofissional de nível superior que realiza o direcionamento do atendimento. Usuários com síndrome gripal sem febre e sem sintomas de gravidade são avaliados pelo enfermeiro. Usuários com febre e/ou sintomas de gravidade são encaminhados direto para avaliação médica. $\mathrm{Na}$ consulta de enfermagem, o enfermeiro notifica os casos e orienta usuários e acompanhantes para isolamento domiciliar por 14 dias do início dos sintomas. Na consulta médica, o médico estratifica a gravidade, realiza a notificação e em casos leves, faz orientação para o isolamento domiciliar aos usuários e acompanhantes por 14 dias do início dos sintomas.

Em casos de sinais de agravamento do quadro clínico, ou Síndrome Respiratória Aguda Grave (SRAG), é realizada a assistência inicial para a estabilização do caso, em seguida o médico da unidade contata o médico plantonista do hospital de campanha, passando o quadro do usuário e comunicando a transferência. A UBS possui uma ambulância disponível 24 horas para realizar a transferência dos casos graves para o hospital de campanha.

\section{2- Garantia dos serviços de Atenção Básica $(A B)$ :}

As demais unidades da Atenção Básica continuam realizando os atendimentos das demandas agudas não relacionadas à síndrome gripal, vacinas, procedimentos e consultas de pré-natal com horário agendado. O usuário é acolhido individualmente: os casos que apresentam queixas não relacionadas à síndrome gripal são encaminhados para a escuta inicial. Nesta etapa, realiza-se a Classificação de Risco e vulnerabilidade, sendo classificados 
como azul para risco ou vulnerabilidade não aguda, verde para risco e/ou vulnerabilidade baixa, amarelo para risco e/ou vulnerabilidade intermediária e vermelho para risco e/ou vulnerabilidade alta. Os casos classificados como azul são orientados a procurar a unidade quando estiver retornado o agendamento. Os casos agudos devem ser atendidos usando as precauções padrão.

Foram reorganizadas as agendas dos profissionais: o enfermeiro e o médico agendam somente gestantes, disponibilizando horário ampliado na agenda para o atendimento das demandas espontâneas agudas, sendo responsáveis também pelas orientações por telefone e/ou visita domiciliar quando necessária. Os usuários em isolamento domiciliar que foram atendidos pelo DISKCORONAVIRUS devem obrigatoriamente receber o primeiro atendimento por telefone pelo médico da sua unidade. Os medicamentos prescritos são entregues no domicílio pelo ACS.

Os atendimentos odontológicos foram reordenados, com o atendimento estruturado, momentaneamente, somente para os usuários que apresentam urgências odontológicas a fim de evitar a aglomeração na sala de espera e reduzir a exposição ao risco dos profissionais e usuários. Os odontólogos e os profissionais da equipe multiprofissional participam do acolhimento e do monitoramento clínico dos casos de síndrome gripal.

Os ACS realizam escala de revezamento no "posso ajudar". Quando necessário realizam visita domiciliar, priorizando os grupos de risco e realizam visita de monitoramento dos casos de síndrome gripal.

A vacinação está funcionando com distribuição de senhas com horário, assim o usuário só se direciona à UBS na hora indicada. A vacinação da Influenza foi realizada em domicílio e em esquema drive thru. Os demais procedimentos realizados na UBS, como teste do pezinho, curativos, administração de medicamentos e retirada de pontos, continuam sendo realizados utilizando as medidas de precauções padrão.

Em relação aos usuários crônicos, cada equipe é responsável por avaliar caso a caso, de acordo com o risco e a vulnerabilidade de cada usuário, a necessidade de atendimento, que caso seja necessário, é realizado no domicílio seguindo as recomendações do Ministério da Saúde em relação às visitas domiciliares.

\section{2 - Elaboração ou readequação de novos serviços para o fortalecimento da RAS \\ 2.1 - Diskcoronavirus}

O Diskcoronavírus é canal de comunicação 0800 entre a população e os profissionais de saúde, que pode ser acessado pelos usuários por meio de ligação ou WhatsApp, sete dias por semana das $8 \mathrm{~h}$ às $20 \mathrm{~h}$. O serviço conta com profissionais capacitados para realizar as orientações iniciais e os encaminhamentos para a UBS de referência dos sintomáticos respiratórios, ou para o Centro de Referência. Quando necessário, os profissionais também realizam a notificação dos casos.

\section{2 - Central de Vigilância e monitoramento clínico dos sintomáticos respiratórios}

Foi montada uma Central de Vigilância, que recebe a notificação de todos os serviços públicos e privados, inclusive do Diskcoronavirus. As notificações são separadas de acordo com o endereço do usuário e são enviadas online para casa UBS de acordo com o território em que o usuário notificado reside.

As unidades básicas realizam o monitoramento por telefone a cada 24 horas ou 48 horas de acordo com o quadro clínico dos usuários. 0 monitoramento clínico é realizado pelo odontólogo e pela equipe multiprofissional.

Caso necessário, o médico e o enfermeiro realizam as orientações por telefone e o ACS entrega a prescrição no domicílio. Quando necessário, o atendimento presencial é realizado pela visita domiciliar. Os ACS também entregam em domicílio o termo de isolamento e as orientações para os usuários. Todos os 
usuários monitorados realizam teste rápido para COVID-19 no domicílio a partir do oitavo dia de sintomas.

Também foi montado um monitoramento clínico exclusivo para os profissionais de saúde e usuários com comorbidades, a equipe é composta por médico, enfermeiro e odontólogo que realizam 0 monitoramento por teleatendimento a cada 24 horas e presencial para avaliação clínica quando necessário, com intuito de prestar um cuidado mais equânime e próximo, a fim de identificar precocemente os sinais de agravamento do caso.

\section{3 - Testagem domiciliar para sintomáticos respiratórios}

Canaã dos Carajás é um dos municípios que mais realiza testes rápidos no estado do Pará. Foi montada uma equipe treinada composta por técnicos de enfermagem, enfermeiros, odontólogos, ACS e equipe multiprofissional, com todos profissionais capacitados para a realização do teste rápido. Todas as pessoas que estão sendo monitoradas, independentemente da gravidade dos sintomas, são testadas a partir do oitavo dia de sintomas no domicílio. Os profissionais de saúde e de segurança e os seus contatos domiciliares que apresentarem sintomas serão testados após o oitavo dia de sintoma e mais três dias sem sintomas. Em alguns casos, os profissionais podem ser testados mesmo que não apresentem toda a clínica de síndrome gripal.

\section{4 - Serviços de ambulância}

A UBS de referência para os sintomáticos respiratórios possui uma ambulância disponível 24 horas para realizar a transferência dos casos graves para o hospital de campanha.

\section{3 - Reorganização da assistência hospitalar \\ 3.1 - Hospital de campanha}

A UBS de referência para os sintomáticos respiratórios possui uma ambulância disponível 24 horas para realizar a transferência dos casos graves para o hospital de campanha.

\section{2 - Hospital da cidade}

O hospital municipal possui 51 leitos. Com a implantação da UBS de referência para sintomáticos respiratórios funcionando 24 horas e a inauguração do hospital de campanha, o fluxo de usuários com sintomas respiratórios que procuram o pronto socorro do hospital diminuiu. O hospital continua realizando os atendimentos das urgências e emergências não relacionadas à COVID-19, incluindo internação clínica, cirúrgica, obstétrica e pediátrica.

\section{4 - Ações intersetoriais}

- Contratação de mais profissionais para ampliar a assistência dos usuários. Todos os profissionais foram capacitados e contam com todos os Equipamentos de Proteção Individual (EPI) necessários para a sua proteção. O município também cumpre todos os protocolos de isolamento e testagem dos profissionais de saúde que apresentam sintomas respiratórios.

- Foram instituídas medidas de distanciamento social ampliado, seguido de alguns dias de lockdown. As medidas de distanciamento são reavaliadas diariamente com intuito de conter a disseminação da COVID-19

- Foram implantadas medidas de precaução comunitária, como pias para lavagem das mãos nas ruas e cabines de desinfecção.

- Ampliação da comunicação com a população através das mídias digitais com a divulgação diária do boletim de casos de COVID-19.

\section{CONCLUSÃO}

Diante do cenário de pandemia fica evidente a necessidade de reorganizar a rede de Atenção em Saúde para ampliar e garantir o acesso da população ao SUS. O município de Canaã dos Carajás reorganizou a RAS, garantindo o acesso e ampliação da carteira de serviços da APS, instituindo uma porta de entrada 24 horas para os sintomáticos respiratórios e a garantia dos serviços básicos da Atenção Básica ( $A B$ ). Também foram elaborados novos serviços, 
readequação dos serviços existentes para o fortalecimento da RAS, além da implantação do Diskcoronavírus, da Central de Vigilância, do monitoramento e testagem domiciliar para sintomáticos respiratórios e da readequação dos serviços de ambulância. Houve a reorganização da Assistência Hospitalar com implantação do hospital de campanha e o fortalecimento das ações do Hospital Municipal. Os processos de planejamento, monitoramento, avaliação e reorganização das RAS devem ser contínuos durante a pandemia, sempre levando em conta as evidências epidemiológicas locais e a orientação comunitária para traçar novas estratégias para garantia de um sistema de saúde universal e equânime. 


\section{NOTAS E REFERÊNCIAS}

1.Conselho Nacional de Secretários de Saúde - CONASS. Guia Orientador para o enfrenta- mento da pandemia Covid-19 na Rede de Atenção à Saúde. BRASÍLIA MAIO DE 2020.

2.TURCI, M.; HOLLIDAY, J.; DE OLIVEIRA, N. A Vigilância Epidemiológica diante do Sars-Cov-2: desafios para o SUS e a Atenção Primária à Saúde. APS EM REVISTA, v. 2, n. 1, p. 44-55, 15 abr. 2020.

3.MINISTÉRIO DA SAÚDE. Portaria no 454. Brasília, 20 de março de 2020

4.GARCIA, Leila Posenato; DUARTE, Elisete. Intervenções não farmacológicas para o enfrentamento à epidemia da COVID-19 no Brasil. Epidemiol. Serv. Saúde, Brasília, v. 29, n. 2, e2020222, 2020.

5.ABREU, Luiz Carlos. Ações integradas e o fortalecimento do Sistema Público de Saúde Brasileiro em tempos de pandemias. Revista brasileira de desenvolvimento humano, n 30 (1), 2020.

6.Ministério da Saúde. Protocolo de manejo clínico do coronavírus (COVID-19) na Atenção Primária à Saúde - Versão 09- Brasília, abril 2020.

7.CEDAPS, FUNDAÇÃO VALE, UNESA. Ciclo Saúde: atores e cenários. In: Ciclo Saúde: construção compartilhada de conhecimentos e ações pelo fortalecimento da Atenção Básica em municípios brasileiros - Ciclo Saúde / Fundação Vale/ CEDAPS /UNESA - Rio de Janeiro: 2017.

8.LIMA, Débora Garcia. "Sistematização da experiência" de reorganização do acolhimento aos usuários nas unidades de atenção básica do município de Canaã dos Carajás, Pará. Rio de janeiro, 2019. 